\title{
Prediction on Deflection of Z-Core Sandwich Panels in Weak Direction
}

\author{
Chen Cheng*
}

School of Civil \& Environmental Engineering, Nanyang Technological University, Nanyang Avenue, Singapore 639798

\begin{abstract}
In a Z-core sandwich panel, the shear stiffness of the sandwich panel in the weak direction which is perpendicular to the placement of the Z-cores is much smaller than the bending stiffness because the hollow section between the two facing plates cannot sustain shear action. Because of this fact, the shear deformation of a Z-core sandwich panel under bending in the weak direction cannot be ignored. Although the flexural deformation of a Z-core sandwich panel can be calculated simply from beam theory, the shear deformation is much more difficult to calculate due to the mutual action between the facing plates and the Z-core stiffener. Considering the contact between the Z-core flanges and the facing plates, the shear deformation of a typical segment in a Z-core sandwich panel is analyzed based on compatibility conditions. By using Castigliano's second theorem, the equations for calculating the deflection caused by shear action of a Zcore sandwich panel under bending in weak direction are obtained. The overall deflection of the Z-core sandwich panel is superposed by the deflections caused by flexural and shear actions respectively. The accuracy of the presented equations for calculating the deflection of a Z-core sandwich panel is finally verified by comparing the predicted results with experimental results reported in corresponding reference. It is found that the predicted results from the presented equation agree quite well with experimental results, which shows the reliability and accuracy of the proposed equations.
\end{abstract}

Keywords: Z-core sandwich panel, bending behavior, weak direction, shear deflection, compatibility conditions.

\section{INTRODUCTION}

A steel sandwich panel is consisted of two facing plates and a core between them. This structure has high flexural stiffness, light weight, and especially it has high resistance to dynamic and blast loads. Due to these advantages, it is widely used in ship and offshore engineering, such as ship and offshore platform decking, or double-skin vessels. It is also potential for this structure to be used in building and constructional industries. Combing with light weight materials, such as aluminum, the sandwich panels can be also used in terrestrial and space engineering.

For a steel sandwich panel, different types are classified according to the cores. Such commonly used types include web-core, $\mathrm{C}$-core, Z-core, X-core, V-core and truss-core etc. The cores are placed generally in a single direction, which causes the sandwich panel of different stiffnesses in the two length directions. In the direction of the core placement, the bending stiffness, the shear stiffness and the torsion stiffness are all high. However, in the direction perpendicular to the core placement, or so-called transverse direction, the shear stiffness is much weaker. The difference of the mechanical properties of a sandwich panel in two directions makes it difficult to analyze this structure theoretically because it is essential to be a composite structure. In the literature, many efforts have been made to simplify a sandwich panel to be an equivalent orthotropic thick plate based on providing the appropriate solutions of some fundamental structural

*Address correspondence to this author at the School of Civil \& Environmental Engineering, Nanyang Technological University, Nanyang Avenue, Singapore 639798; Tel: +6565923074 Fax: +6583847546

E-mail: ChenChang@ntu.edu.sg properties. Such early representative work was conducted by Libove and Batforf (1948) [1], and Libove and Hubka (1951) [2]. Simplifying a 3D sandwich panel into a $2 \mathrm{D}$ equivalent plate, it is necessary to give accurate solution of some elastic constants such as the bending stiffnesses in two directions, the twisting stiffnesses and the shear stiffnesses in two directions. Libove and Hubka (1951) derived the elastic constants for sandwich panels with a continuous corrugated core based on the assumption that the cross section of the core-stiffeners is symmetrical about a vertical plane. Later on, many researchers paid much effort on deriving accurate solutions of these elastic constants, such as the work reported in Refs. [3-8]. Based on these closed-form solutions of the elastic constants, the static behavior of sandwich panels can be analyzed theoretically and numerically. Cheng et al. (2006) [9] evaluated the accuracy of some elastic constants of sandwich panels with various cores by using finite element analysis. Chang et al. (2005) [10] studied the bending behavior of corrugated-core sandwich panels by using Mindlin-Reissner plate theory. Romanoff et al. (2006, 2007) [11-13] analyzed the bending behavior of web-core sandwich beams by using the solutions of elastic constants. Buannic et al. (2003) [14] also investigated the behavior of corrugated core sandwich panels by using finite element method.

In this paper, Z-core sandwich panel under bending in weak direction is studied. The bending behavior of the Zcore sandwich panel is then assessed from the load versus deflection relationship. In calculating the deflection, a simplified theoretical analysis for deriving the shear deformation is conducted, and closed form equations for calculating the deflection of Z-core sandwich panels are presented. The accuracy of the derived equations is also verified. 


\section{THEORETICAL ANALYSIS}

\section{Typical Segment in a Z-Core Sandwich Panel}

As shown in Fig. (1), a Z-core sandwich panel is consisted of a series of Z-core stiffeners and two facing plates, which are called top facing plate and bottom facing plate respectively. The Z-core stiffeners are connected to the top and bottom facing plates by using laser spot weld or selftapping screws. Generally, the Z-core stiffeners are placed only in one direction, as it is shown in Fig. (1) that the Zcore stiffeners are placed along $\mathrm{x}$-axis. Due to this reason, the sandwich panel has different stiffnesses in $\mathrm{x}$ - and $\mathrm{y}$ directions. It is easy to see that the direction in y-axis is the weak direction due to lack of strengthening of the Z-core. As the facing plates are mainly subjected to bending action and the Z-core stiffeners are used to resist the shear action, the shear stiffness in the weak direction is definitely weaker.

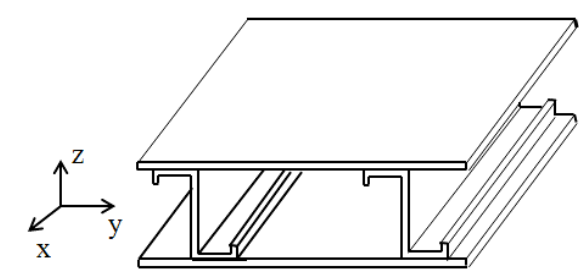

Fig. (1). A C-core sandwich panel.

If a Z-core sandwich panel is subjected to uniform loading, a typical segment as shown in Fig. (2) can be isolated to analyze. It is assumed that each segment in a Z-core sandwich panel has similar deformation property to its adjacent one, or each segment has same mechanism in deforming. This assumption makes it reasonable that a representative typical segment as shown in Fig. (2) can be studied, and the deformation calculation of this segment can be also applicable for other segments. In Fig. (2), the length of the top and the bottom facing plates of the segment is denoted by $s$. This length is also identical to the distance between any two adjacent C-cores. The distance between the mid-plane of the top and bottom facing plates is denoted by $h_{\mathrm{p}}$. Similarly, $h_{\mathrm{c}}$ is used to represent the distance between the mid-planes of the two flanges of the Z-core. The web of the Z-core stiffener is located in the mid-point of the facing plates, and the position of the connection between the facing plates and the Z-core are described by $l_{\mathrm{a}}, l_{\mathrm{b}}, l_{\mathrm{c}}$ and $l_{\mathrm{d}}$ respectively. It is noted that the segment is still itself if it is rotated by $180^{\circ} l_{\mathrm{a}}=l_{\mathrm{b}}$ and $l_{\mathrm{c}}=$ $l_{\mathrm{d}}$. However, the segment in Fig. (2) represents a more general model. For brevity yet more practical, the thicknesses of the top and the bottom facing plates are assumed to be same, and it is denoted by $t_{\mathrm{p}}$. The thickness of the Z-core, which has a constant value, is denoted by $t_{\mathrm{z}}$.

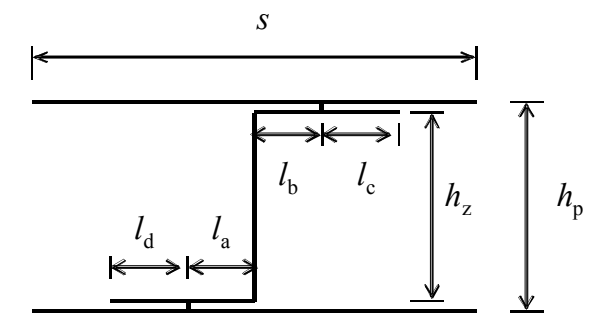

Fig. (2). Typical segment in C-core sandwich panel.
If a Z-core sandwich panel is subjected to bending load, the panel has no axial forces in any cross section in thickness direction. Therefore, only shear force and bending moment exist in the cross section. In the weak direction, the bending moment is assumed to be replaced by a couple of forces at the two facing plates, as shown in Fig. (3). However, the bending moments at the left and the right ends of the segment may be different in general case. It is assumed that the bending moments at the left end and at the right end are $M$ and $M+\Delta M$ respectively. $M$ is replaced by an equivalent couple of forces $N$ and $M+\Delta M$ is replaced by a couple of forces $N+\Delta N$. The following equation can be obtained

$$
\left\{\begin{array}{l}
M=N h_{p} \\
M+\Delta M=(N+\Delta N) h_{p}
\end{array}\right.
$$
ists

Eq. (1) also indicates that the following relationship ex-

$$
\Delta M=\Delta N \times h_{p}
$$

The shear forces at two ends of the segment, however, are same. The shear force at each end is sustained by the top and the bottom facing plates. It is assumed that the shear force at each end is $V$. If the directions of the shear forces at two ends of the segment are defined as shown in Fig. (3), contact will exist at the ends of the flange of the Z-core stiffener. The contact forces are denoted by $F_{\mathrm{c} 1}$ and $F_{\mathrm{c} 2}$ respectively. Due to the contact problem, the shear force sustained by the top facing plate is not equal to that sustained by the bottom facing plate. It is assumed that the shear forces distributed to the top and the bottom facing plates are $V / 2-\Delta V$ and $V / 2+\Delta V$ respectively. To make the segment in equilibrium, the following equation must be satisfied

$$
(V / 2-\Delta V) \times s+(V / 2+\Delta V) \times s=\Delta M
$$

Eq. (3) can be simplified to the following equation

$$
V s=\Delta N h_{p}
$$
tion

Then $\Delta N$ can be determined from the following equa-

$$
\Delta N=V_{S} / h_{p}
$$

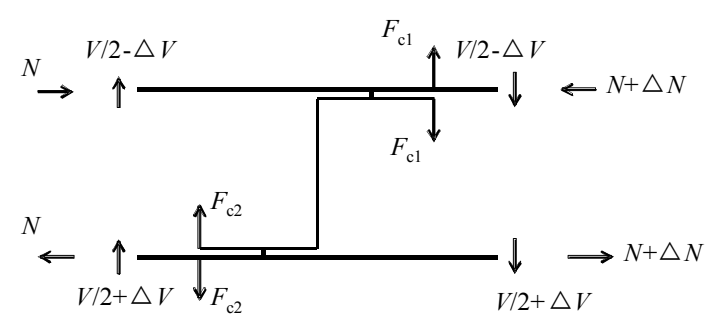

Fig. (3). Equilibrium of segment in weak arrangement.

However, the contact points are different if the shear forces at the two ends are defined as shown in Fig. (4). Fig. (5a) and Fig. (5b) shows the different deformations when the shear forces at two ends of the segment are different. The segment has bigger stiffness if it deforms as shown in Fig. (5b), and such it is called strong arrangement. Accordingly, Fig. (5a) shows a weak arrangement of the segment. Such 
definitions on the strong and weak arrangements are also given by Fung and Tan (1998). The equilibriums of the typical segment shown in Figs. (3) and (4) are in accordance with the deformation in Figs. (5a) and (5b) respectively.

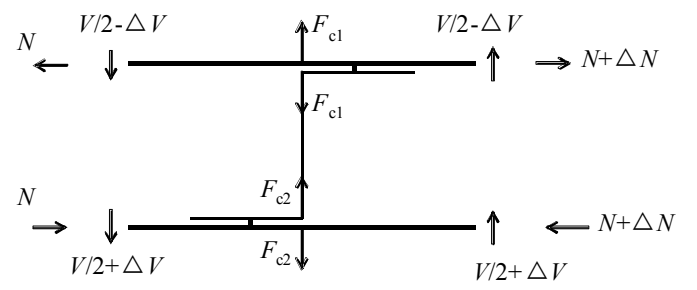

Fig. (4). Equilibrium of segment in strong arrangement.

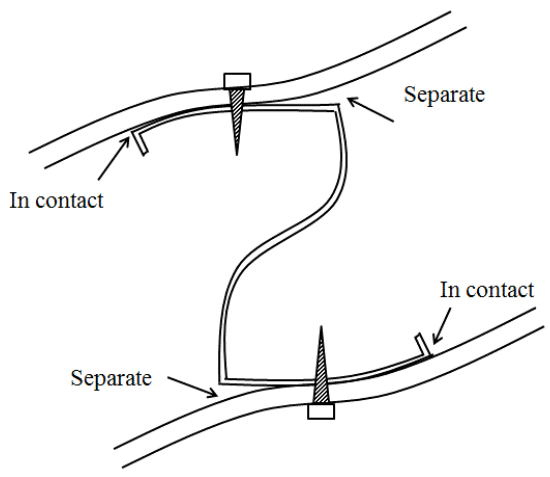

(a) weak arrangement

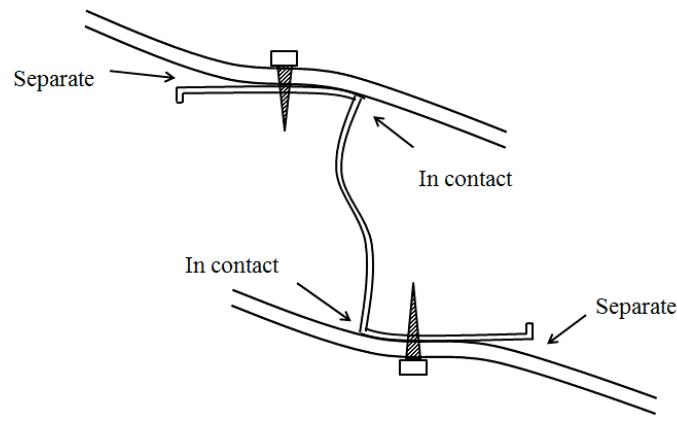

(b) strong arrangement

Fig. (5). Definition of weak and strong arrangement.

If the shear force $V$ and the bending moments $M$ and $M+\Delta M$ are known, only three unknowns in the segment shown in Fig. (3) or in Fig. (4) are necessary to be calculated, and they are $F_{c 1}, F_{c 2}$ and $\Delta V$. The closed-form solutions of the three unknowns should be obtained from compatibility conditions between adjacent segments.

\section{Compatibility Conditions Between Adjacent Segments}

The compatibility conditions of the typical segment in weak arrangement can be shown in Fig. (6).

The following two assumptions are made:

(1) There is no relative displacement between the facing plate and the Z-core flange at contact points. This assumption is meaningful since there will be no separation between any contact points due to compression action. This assumption will produce the following two equations:

$$
\begin{aligned}
& \Delta_{p 1}+\Delta_{p 2}=0 \\
& \Delta_{Q 1}+\Delta_{Q 2}=0
\end{aligned}
$$

(2) The relative displacement between the top facing plate and the bottom facing plate should be equal at the two ends of the segment. The following equation must be satisfied according to this assumption

$$
\Delta_{2}-\Delta_{1}=\Delta_{3}-\Delta_{4}
$$

For the segment in strong arrangement, similar compatibility conditions can be assumed except the positions of the contact points are different.

Eqs. (6)-(8) can be used to derive the solutions of the three unknowns $F_{c 1}, F_{c 2}$ and $\Delta V$.

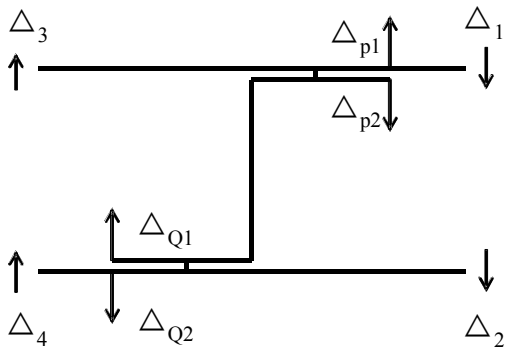

Fig. (6). Compatibility conditions.

\section{Compatibility Equations}

As can be seen from Eqs. (6)-(8), the compatibility conditions are essentially to calculate the relative displacement at some critical positions. This can be done by using Castigliano's second theorem. To do so, the moment diagram of the segment in equilibrium as shown in Figs. (3)-(4) is firstly drawn. For brevity, the bending moment $M_{\mathrm{t}}$ can be divided into several parts:

$$
M_{t}=M_{a}(V, \Delta N)+M_{b}(\Delta V)+M_{c}(N)+M_{d}\left(F_{c 1}, F_{c 2}\right)
$$

Where $M_{\mathrm{a}}(V, \Delta N), M_{\mathrm{b}}(\Delta V), M_{\mathrm{c}}(N)$ and $M_{\mathrm{d}}\left(F_{\mathrm{c} 1}, F_{\mathrm{c} 2}\right)$ are the moments caused by different loadings.

\section{Weak Arrangement}

The moment diagrams produced by $M_{\mathrm{a}}(V, \Delta N), M_{\mathrm{b}}(\Delta V)$, $M_{\mathrm{c}}(N)$ and $M_{\mathrm{d}}\left(F_{\mathrm{c} 1}, F_{\mathrm{c} 2}\right)$ for the Z-core sandwich segment in weak arrangement are shown in Figs. (7a)-(7d) respectively. In each moment diagram from Fig. (7a) to (7d), the segment is always in equilibrium.

In Eq. (6), to calculate $\Delta_{p 1}+\Delta_{p 2}$, the moment diagram as shown in Fig. (8) is generated. Two unit loads in this figure are applied at the contact point between the top facing plate and the top Z-core flange. Then $\Delta_{p 1}+\Delta_{p 2}$ can be calculated from the following equation

$$
\Delta_{p 1}+\Delta_{p 2}=\int_{\Gamma} \frac{M_{t}}{E I} M_{d}(1,0) d \Gamma
$$

where $M_{d}(1,0)$ means the moment diagram by letting $F_{c 1}=1$ and $F_{c 2}=0 ; E$ is the elastic modulus of the steel material, $I$ is the second moment of area about the mid-plane. 


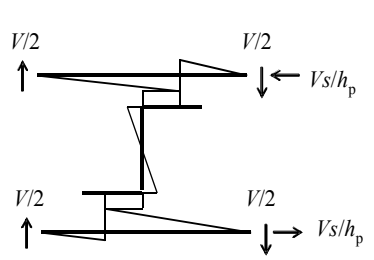

(a) $M_{\mathrm{a}}(V, \Delta N)$

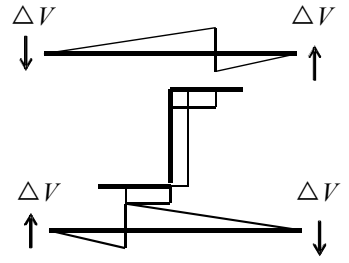

(b) $M_{\mathrm{b}}(\Delta V)$

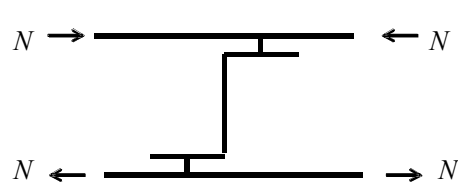

(c) $M_{\mathrm{c}}(N)$

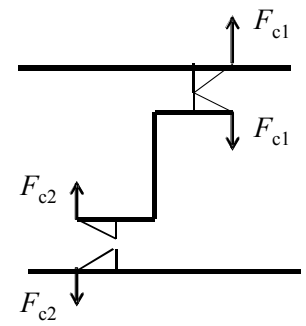

(d) $M_{\mathrm{d}}\left(F_{\mathrm{c} 1}, F_{\mathrm{c} 2}\right)$

Fig. (7). Moment diagrams of segment in weak arrangement.

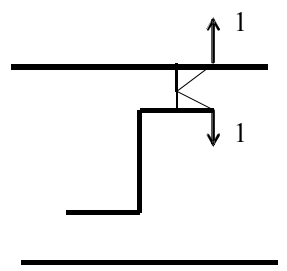

Similarly, using the moment diagram $M_{b}(1)$ as shown in Fig. (10), the following equation can be obtained as well

$$
\Delta_{2}-\Delta_{1}-\Delta_{3}+\Delta_{4}=\int_{\Gamma} \frac{M_{t}}{E I} M_{b}(1) d \Gamma
$$

Substituting the above equation into Eq. (14), the following equation can be obtained

Fig. (8). Moment diagram.

Substituting Eq. (10) into compatibility condition Eq. (6), the following equation can be obtained

$$
F_{c 1}=\frac{\beta}{1+\beta} \frac{3}{2 l}\left[\left(\frac{V}{2}-\Delta V\right)\left(\frac{s}{2}-l_{b}-\frac{l_{c}}{3}\right)\right]
$$

where $\beta=E_{z} I_{z} / E I$ is the ratio of bending stiffness of the Z-core stiffener and the facing plates about their respective mid-plane in thickness direction, i.e. for unit width plate along $x$-axis, $I_{z}=t_{z}^{3} / 12$ and $I=t_{p}^{3} / 12$.

Similarly, the compatibility condition at the contact point between the bottom facing plate and the bottom Z-core flange can be calculated from the following equation

$$
\Delta_{Q 1}+\Delta_{Q 2}=\int_{\Gamma} \frac{M_{t}}{E I} M_{d}(0,1) d \Gamma
$$

where $M_{d}(0,1)$ is shown in Fig. (9).

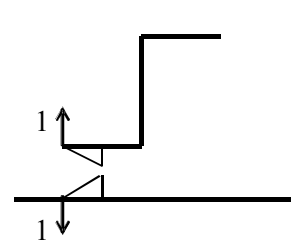

Fig. (9). Moment diagram $M_{d}(0,1)$.

From Eq. (7), the following equation can be obtained

$$
F_{c 2}=\frac{\beta}{1+\beta} \frac{3}{2 l_{d}}\left[\left(\frac{V}{2}+\Delta V\right)\left(\frac{s}{2}-l_{a}-\frac{l_{d}}{3}\right)\right]
$$

Compatibility condition Eq. (8) can be transformed into the following format

$\Delta_{2}-\Delta_{1}-\Delta_{3}+\Delta_{4}=0$

$\Delta V=\frac{\left(-\alpha_{8}+\alpha_{9}\right) \times \frac{V}{2}}{\alpha_{1}+\alpha_{2}+\alpha_{3}+\alpha_{4}+\alpha_{5}+\alpha_{6}+\alpha_{7}-\alpha_{8}-\alpha_{9}}$

where $\quad \alpha_{1}=\frac{\left(\frac{s}{2}+l_{b}\right)^{3}}{3} ; \quad \alpha_{2}=\frac{\left(\frac{s}{2}-l_{b}\right)^{3}}{3} ; \quad \alpha_{3}=\frac{\left(\frac{s}{2}-l_{a}\right)^{3}}{3}$;

$\alpha_{4}=\frac{\left(\frac{s}{2}+l_{a}\right)^{3}}{3} ; \quad \alpha_{5}=\frac{l_{b} s^{2}}{\beta} ; \quad \alpha_{6}=\frac{l_{a} s^{2}}{\beta} ; \quad \alpha_{7}=\frac{h_{z} s^{2}}{\beta} ;$

$\alpha_{8}=\frac{3 l_{c}}{4} \frac{\beta}{1+\beta}\left(\frac{s}{2}-l_{b}-\frac{l_{c}}{3}\right)^{2} ; \alpha_{9}=\frac{3 l_{d}}{4} \frac{\beta}{1+\beta}\left(\frac{s}{2}-l_{a}-\frac{l_{d}}{3}\right)^{2}$

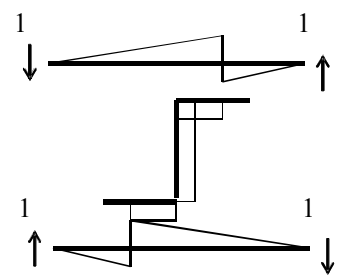

Fig. (10). Moment diagram.

From Eqs. (11), (13) and (16), it is easy to find that the three unknowns $F_{c 1}, F_{c 2}$ and $\Delta V$ can be calculated once the value of shear force $V$ at two ends of the segment is known. The steps can be generalized as follows

(1) From Eq. (16), the value of $\Delta V$ can be calculated when the value of shear force $V$ is known.

(2) Substituting the values of $V$ and $\Delta V$ into Eqs. (11) and (13), the values of $F_{c 1}$ and $F_{c 2}$ can be obtained.

\section{Strong Arrangement}

Using the same method, the equations for calculating the three unknowns $F_{c 1}, \quad F_{c 2}$ and $F_{c 1}=\frac{\beta}{1+\beta} \frac{3}{2 l_{b}}\left[\left(-\frac{V}{2}+\Delta V\right)\right.$ 
$\left.\mathrm{I}\left(\frac{s}{2}+\frac{2 l_{b}}{3}\right)-\frac{V s h_{z}}{2 \beta h_{p}}+\frac{\Delta V s}{\beta}\right]$ can also be obtained. The detailed calculating process is not repeated, and the derived equations are listed as follows

$$
\begin{aligned}
& F_{c 1}=\frac{\beta}{1+\beta} \frac{3}{2 l_{b}}\left[\left(-\frac{V}{2}+\Delta V\right)\left(\frac{s}{2}+\frac{2 l_{b}}{3}\right)-\frac{V s h_{z}}{2 \beta h_{p}}+\frac{\Delta V s}{\beta}\right] \\
& F_{c 2}=\frac{\beta}{1+\beta} \frac{3}{2 l_{a}}\left[-\left(\frac{V}{2}+\Delta V\right)\left(\frac{s}{2}+\frac{2 l_{a}}{3}\right)-\frac{V h_{z}^{2}}{2 h_{p}}-\frac{\Delta V s}{\beta}\right] \\
& \Delta V=\frac{\left(-\alpha_{8}-\alpha_{9}+\alpha_{11}+\alpha_{12}\right) \times \frac{V}{2}+\left(-\frac{\alpha_{9}}{2}-\alpha_{10}+\alpha_{13}+\alpha_{14}\right) \frac{V h_{z}}{h_{p}}}{\alpha_{1}+\alpha_{2}+\alpha_{3}+\alpha_{4}+\alpha_{5}+\alpha_{6}+\alpha_{7}-\alpha_{8}-2 \alpha_{9}-\alpha_{10}-\alpha_{11}-2 \alpha_{12}-\alpha_{13}}
\end{aligned}
$$
follows:

In Eq. (19), the expressions of the constants are listed as

$$
\begin{aligned}
& \alpha_{1}=\frac{\left(\frac{s}{2}+l_{b}\right)^{3}}{3} ; \quad \alpha_{2}=\frac{\left(\frac{s}{2}-l_{b}\right)^{3}}{3} ; \quad \alpha_{3}=\frac{\left(\frac{s}{2}+l_{a}\right)^{3}}{3} ; \quad \alpha_{4}=\frac{\left(\frac{s}{2}-l_{a}\right)^{3}}{3} ; \\
& \alpha_{5}=\frac{l_{b} s^{2}}{\beta} ; \quad \alpha_{6}=\frac{l_{a} s^{2}}{\beta} ; \quad \alpha_{7}=\frac{h_{z} s^{2}}{\beta} ; \quad \alpha_{8}=\frac{\beta}{1+\beta} \frac{3 l_{b}}{4}\left(\frac{s}{2}+\frac{2 l_{b}}{3}\right)^{2} \\
& \alpha_{9}=\frac{1}{1+\beta} \frac{3 l_{b} s}{4}\left(\frac{s}{2}+\frac{2 l_{b}}{3}\right) ; \alpha_{10}=\frac{1}{1+\beta} \frac{3 l_{b} s^{2}}{4 \beta} ; \alpha_{11}=\frac{\beta}{1+\beta} \frac{3 l_{a}}{4}\left(\frac{s}{2}+\frac{2 l_{a}}{3}\right)^{2} \\
& \alpha_{12}=\frac{1}{1+\beta} \frac{3 l_{a} s}{4}\left(\frac{s}{2}+\frac{2 l_{a}}{3}\right) ; \alpha_{13}=\frac{\beta}{1+\beta} \frac{3 l_{a} h_{z}}{8}\left(\frac{s}{2}+\frac{2 l_{a}}{3}\right) ; \alpha_{14}=\frac{1}{1+\beta} \frac{3 l_{a} s h_{z}}{8}
\end{aligned}
$$

\section{Shear Deformation in Vertical Direction}

After the values of the unknowns can be calculated, the shear deformation of the segment shown in Figs. (3) and (4) can be analyzed. To calculate a Z-core sandwich panel under bending, only the deformation in vertical direction is analyzed, which is shown in Fig. (11). The shear deformation in vertical direction, $\Delta_{\mathrm{s}}$, of this segment is calculated from the following equation

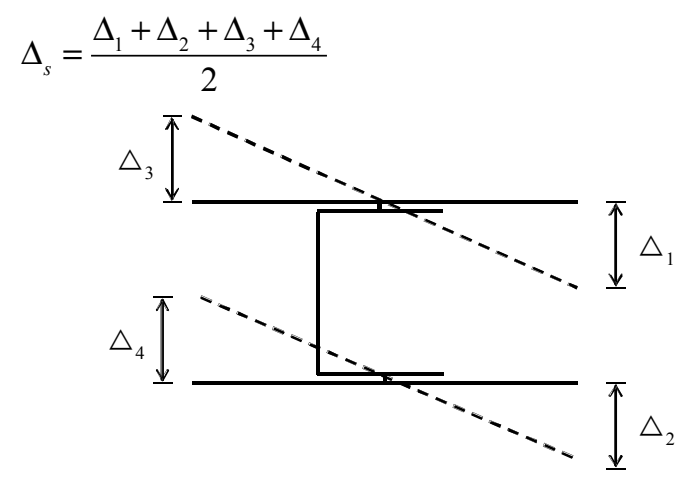

Fig. (11). Shear deformation of a segment.

To calculate $\Delta_{\mathrm{s}}$, a moment diagram $\bar{M}$ as shown in Fig. (12) is generated. Then $\Delta_{\mathrm{S}}$ is obtained from the following equation

$$
\Delta_{s}=\frac{1}{2} \int_{\Gamma} \frac{M_{t}}{E I} \bar{M} d \Gamma
$$

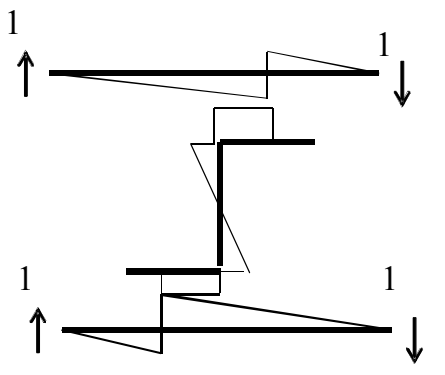

Fig. (12). Moment diagram $\bar{M}$.

Combing with Eqs. (11), (13) and (16), the vertical displacement $\Delta_{\mathrm{s}}$ caused by shear action for the Z-core sandwich panel in weak arrangement can be calculated from the following equation

$$
\begin{aligned}
& \Delta_{s}=\frac{V}{12 E I}\left[\begin{array}{l}
\left(\frac{s}{2}+l_{b}\right)^{3}+\left(\frac{s}{2}-l_{b}\right)^{3}+\left(\frac{s}{2}+l_{a}\right)^{3}+\left(\frac{s}{2}-l_{a}\right)^{3} \\
+\frac{3(a+b) s^{2} h_{z}}{\beta h}+\frac{s^{2} h_{z}^{2}}{\beta h}
\end{array}\right] \\
& -\frac{\Delta V}{6 E I}\left[\left(\frac{s}{2}+l_{b}\right)^{3}+\left(\frac{s}{2}-l_{b}\right)^{3}-\left(\frac{s}{2}+l_{a}\right)^{3}-\left(\frac{s}{2}-l_{a}\right)^{3}\right] \\
& -\frac{1}{4 E I}\left[F_{c 1} l_{c}^{2}\left(\frac{s}{2}-l_{b}-\frac{l_{c}}{3}\right)+F_{c 2} l_{d}^{2}\left(\frac{s}{2}-l_{a}-\frac{l_{d}}{3}\right)\right]
\end{aligned}
$$

For the Z-core sandwich panel in strong arrangement, the shear deformation can be also obtained in the similar way, and it is listed as follow

$$
\begin{aligned}
& \Delta_{s}=\frac{V}{12 E I}\left[\begin{array}{l}
\left.\left(\frac{s}{2}+l_{b}\right)^{3}+\left(\frac{s}{2}-l_{b}\right)^{3}+\left(\frac{s}{2}+l_{a}\right)^{3}\left(\frac{s}{2}-l_{a}\right)^{3}+\right] \\
\frac{3(a+b) s^{2} h_{z}}{\beta h}+\frac{s^{2} h_{z}^{2}}{\beta h}
\end{array}\right] \\
& +\frac{\Delta V}{6 E I}\left[\left(\frac{s}{2}+l_{b}\right)^{3}+\left(\frac{s}{2}-l_{b}\right)^{3}-\left(\frac{s}{2}+l_{a}\right)^{3}-\left(\frac{s}{2}-l_{a}\right)^{3}\right] \\
& +\frac{1}{4 E I}\left[F_{c 1} l_{b}^{2}\left(\frac{s}{2}+\frac{2 l_{b}}{3}\right)+\frac{F_{c 1} l_{b}^{2} s}{\beta}+F_{c 2} l_{a}^{2}\left(\frac{s}{2}+\frac{2 l_{a}}{3}\right)+\frac{F_{c 2} l_{a}^{2} s}{\beta}\right]
\end{aligned}
$$

If a Z-core sandwich panel is consisted of $n$ typical segments, then the total vertical displacement of the sandwich panel, $w_{\mathrm{S}}$, is calculated from the following equation

$$
w_{s}=n \Delta_{s}
$$

As the facing plates and the Z-core stiffener in bending behave in a plane strain state, the elastic modulus $E$ in all above equations should be replaced by $E^{*}$, and $E^{*}=E /\left(1-v^{2}\right)$, where $v$ is Poisson's ratio.

\section{Bending Deformation in Vertical Direction}

For a Z-core sandwich panel under bending in weak direction, the bending deformation can be calculated simply from beam theory. However, the second moment of area of the Z-core sandwich panel in weak direction, $I_{\mathrm{s}}$, is calculated from the following equation 


$$
I_{s}=\frac{1}{2} t_{p} h_{p}^{2}
$$

Eq. (25) is obtained based on the assumption that the bending moment is sustained by the top and the bottom facing plates while the Z-core stiffeners do not bear any bending moment.

\section{CASE STUDY}

\section{Verification Through Experimental Results}

To verify the presented method in calculating the vertical deformation of Z-core sandwich panels under bending in weak direction, an experimental model reported by Fung and Tan (1998) is used to assess the accuracy of this method. The basic dimensions of the Z-core sandwich panel can be found in Fig. (13). The panel is $495 \mathrm{~mm}$ in width and $2000 \mathrm{~mm}$ in length, with $16 \mathrm{Z}$-sections as core stiffeners at a nominal spacing of $130 \mathrm{~mm}$. The facing plates and the Z-sections are connected through self-taping screws. The other dimensions can be found in Table $\mathbf{1}$.

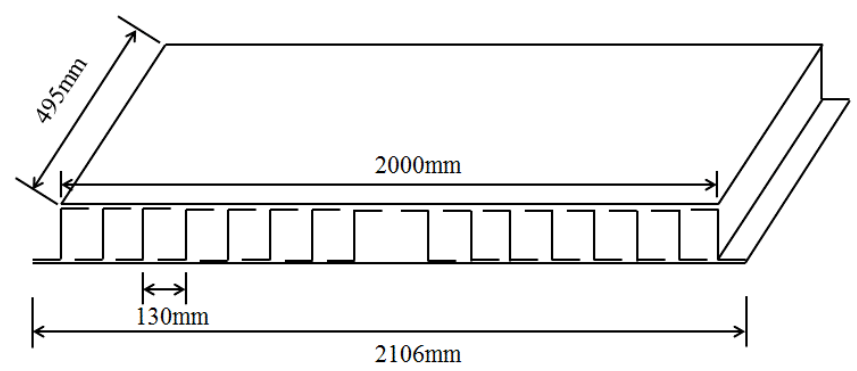

Fig. (13). Z-core sandwich panel specimen.

Table 1. Dimensions of the Z-Core Sandwich Panel

\begin{tabular}{|c|c|}
\hline Parameter & Panel \\
\hline$s(\mathrm{~mm})$ & 130.0 \\
\hline$l_{\mathrm{a}}(\mathrm{mm})$ & 26.5 \\
\hline$l_{\mathrm{b}}(\mathrm{mm})$ & 24.0 \\
\hline$l_{\mathrm{c}}(\mathrm{mm})$ & 24.0 \\
\hline$l_{\mathrm{d}}(\mathrm{mm})$ & 26.5 \\
\hline$h_{\mathrm{p}}(\mathrm{mm})$ & 104 \\
\hline$h_{\mathrm{z}}(\mathrm{mm})$ & 100.0 \\
\hline$v$ & 0.3 \\
\hline$E\left(\mathrm{~N} / \mathrm{mm}^{2}\right)$ & 207500 \\
\hline$E_{\mathrm{c}}\left(\mathrm{N} / \mathrm{mm}^{2}\right)$ & 207500 \\
\hline$t_{\mathrm{p}}(\mathrm{mm})$ & 2.0 \\
\hline$t_{\mathrm{c}}(\mathrm{mm})$ & 2.0 \\
\hline
\end{tabular}

The panel is placed on two roller bearings at the ends to simulate an ideal simply supported boundary conditions. The supporting centres of the rollers are assumed to be at the point just under the web of the Z-core stiffener. The panel is subjected to a line load at the midspan, and thus it is a threepoint bending model, as shown in Fig. (14). In experimental test, this panel was tested in its weak arrangement. The relationship between the applied load and the deflection at the midspan is obtained from test measurement. Fung and Tan (1998) presented the relationship between the applied load $P$ and the deflection at the midspan $w$ as follows

(1) for the first specimen in weak arrangement

$$
P=45.96 w
$$

In Eq. (26), the units of $P$ and $w$ are $\mathrm{N} / \mathrm{mm}$ and $\mathrm{mm}$, or $\mathrm{kN} / \mathrm{m}$ and $\mathrm{m}$ respectively.

(2) for the second panel in strong arrangement

$$
P=193.96 w
$$

The overall deflection of each Z-core sandwich panel under three-point bending is consisted of two parts: the deflection caused by bending $w_{\mathrm{b}}$ and the deflection produced by shear $w_{\mathrm{s}}$. The bending deflection is calculated from the following equation.

$$
w_{b}=\frac{\left(1-v^{2}\right) P L^{3}}{48 E I_{s}}
$$

where $L=2000 \mathrm{~mm}$ and 1110 for the first and the second Z-core sandwich panels respectively.

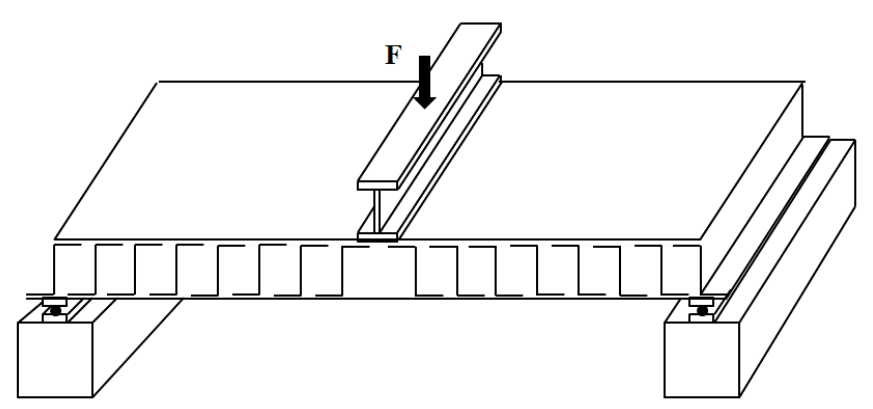

Fig. (14). Loading scheme on the Z-core sandwich panel specimen.

Using Eqs. (23) and (24), the shear deflection $w_{\mathrm{s}}$ can also be calculated. The values of $n$ in Eq. (24) for the two panels are 7.5 and 5.5 respectively. The values of $n$ are not 8 and 6 for the first and for the second panels respectively because the supporting positions at two ends of the panels are located at the web of the Z-core stiffeners, as can be seen in Fig. (14), which makes the end Z-core stiffener only has a half length. The predicted total deflection of the panel, $w$, is the sum of $w_{\mathrm{b}}$ and $w_{\mathrm{s}}$. If a line load with values of $0.928 \mathrm{~N} / \mathrm{mm}$ and $3.92 \mathrm{~N} / \mathrm{mm}$ is applied to the first and the second panels, the deflections at the midspan of the sandwich panels are generalized in Table $2 . w_{\mathrm{e}}$ in Table $\mathbf{2}$ denotes the experimental value of the deflection at midspan. From Table 2, it can be seen that the deflection of the two Z-core sandwich panels is mainly dominant by the shear deformation while the bending deflection is much smaller. The predicted result of the relationship between the load and the displacement at the mid-point of the panel can be calculated from the presented equations, and they are generalized as follows 
(1) for the first panel in weak arrangement

$$
P_{e}=42.56 w
$$

(2) for the second panel in strong arrangement

$$
P_{e}=181.27 w
$$

Table 2. Comparison Between Experimental and Predicted Results

\begin{tabular}{|c|c|c|}
\hline Parameter & Panel 1 & Panel 2 \\
\hline \hline$P(\mathrm{~N} / \mathrm{mm})$ & 0.928 & 3.92 \\
\hline$w_{\mathrm{e}}(\mathrm{mm})$ & 10.0 & 10.0 \\
\hline$w_{\mathrm{b}}(\mathrm{mm})$ & 0.064 & 0.045 \\
\hline$w_{\mathrm{s}}(\mathrm{mm})$ & 10.737 & 10.656 \\
\hline$w(\mathrm{~mm})$ & 10.8 & 10.7 \\
\hline
\end{tabular}

Figs. (15a) and (15b) show the comparison of the load versus displacement of the two panels between predicted and experimental fitting results. The comparison shows the presented equations can provide reasonably good estimation for the bending behaviour of Z-core sandwich panels.

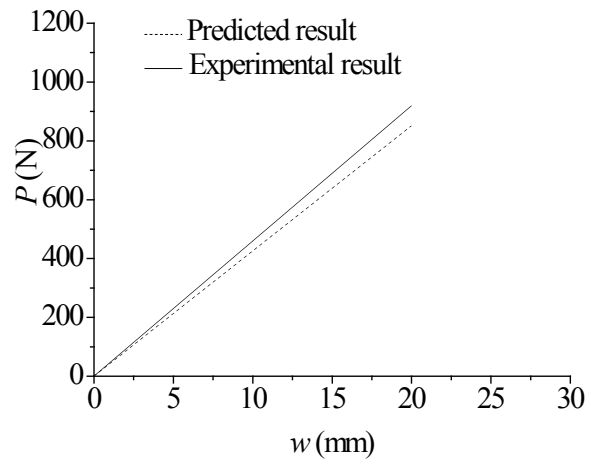

(a) Panel 1 in weak arrangement

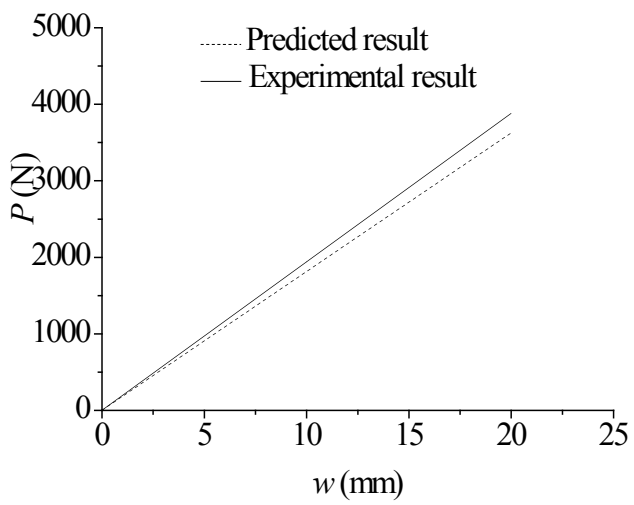

(b) Panel 2 in strong arrangement

Fig. (15). Comparison of the load-displacement relationship for the panel specimens.

A relative error between the results calculated from the theoretical method in this study and the experimental measurement is defined as follow

$$
e=\frac{P_{e}-P}{P} \times 100 \%
$$

From Eq. (31), the calculated relative errors are $-7.4 \%$, and $-6.5 \%$ respectively. The values of the errors for the two models are both negative, and it means the predicted load is smaller than the experimentally measured load. Therefore, the predicted results are safe. It can be seen that the values of the errors are acceptable, and the accuracy and reliability of the presented equations are ensured.

\section{CONCLUSIONS}

The bending behavior of Z-core sandwich panel is studied, and equations for predicting the shear deformation of Zcore sandwich panel in weak direction with weak and strong arrangements are derived. Through case study, the following two conclusions can be made:

1. The presented equations in this study are accurate and reliable in calculating the deflection of Z-core sandwich panel under bending in its weak direction.

2. For Z-core sandwich panel under bending in weak direction, the shear deformation is much bigger than the flexural deformation.

\section{CONFLICT OF INTEREST}

The author(s) confirm that this article content has no conflicts of interest.

\section{ACKNOWLEDGEMENT}

Declared none.

\section{REFERENCES}

[1] C. Libove and S.B. Batdorf, "A general small deflection theory for flat sandwich plates," NACA Tech. Note 1526, Nat. Advisory Com. for Aeronautics, Washingtong D.C., 1948.

[2] C. Libove and R.E. Hubka, "Elastic constants for corrugated-core sandwich plates," NACA Tech. Note 2289, Nat. Advisory Com. for Aeronautics, Washingtong D.C., 1951.

[3] T. Nordstrand, L.A. Carlsson and H.G. Allen, "Transverse shear stiffness of structural core sandwich," Composite Struct., vol. 27, pp. 317-329, 1994

[4] T.C. Fung, K.H. Tan and T.S. Lok, "Shear stiffness $\mathrm{D}_{\mathrm{Qy}}$ for C-core sandwich panels," J. Struct. Eng., ASCE, vol. 122, pp. 958-966, 1996.

[5] T.C. Fung and K.H. Tan, "Shear stiffness for Z-core sandwich panels," J. Struct. Eng., ASCE, vol. 124, pp. 809-816, 1998.

[6] T.C. Fung, K.H. Tan and T.S. Lok, "Elastic constants for Z-core sandwich panels," J. Struct. Eng., ASCE, vol. 120, pp. 3046-3055, 1994.

[7] T.S. Lok and Q.H. Cheng, "Elastic stiffness properties and behavior of truss-core sandwich panel," J. Struct. Eng., ASCE, vol. 126, pp. 552-559, 2000.

[8] P. Kujala and A. Klanac, "Analytical and numerical analysis of non-symmetrical all steel sandwich panels under uniform pressure load. In international design conference - design Dubrovnik, pp. 1205-1210, 2002.

[9] Y.B. Shao, S.T. Lie and S.P. Chiew, "Static strength of tubular Tjoints with reinforced chord under axial compression," Adv. Struct. Eng., vol. 13, pp. 369-378, 2010.

[10] W.S. Chang, E. Ventsel, T. Krauthammer and J. John, "Bending behavior of corrugated-core sandwich plates," Composite Struct., vol. 70, pp. 81-89, 2005.

[11] J. Romanoff and P. Varsta, "Bending response of web-core sandwich beams," Composite Struct., vol. 73, pp. 478-487, 2006. 
[12] J. Romanoff and P. Varsta, "Bending response of web-core sandwich plates," Composite Struct., vol. 81, pp. 292-302, 2007.

[13] J. Romanoff, P. Varsta and A. Klanac, "Stress analysis of homogenized web-core sandwich beams," Composite Struct., vol. 79, pp. 411-422, 2007.
[14] N. Buannic, P. Cartraud and T. Quesnel, "Homogenization of corrugated core sandwich panels," Composite Struct., vol. 59, pp. 299$312,2003$.

Received: September 02, 2013

Revised: October 03, 2013

Accepted: October 06, 2013

(C) Chen Cheng; Licensee Bentham Open.

This is an open access article licensed under the terms of the Creative Commons Attribution Non-Commercial License (http://creativecommons.org/licenses/by-nc/3.0/) which permits unrestricted, non-commercial use, distribution and reproduction in any medium, provided the work is properly cited. 